\title{
FINE NEEDLE ASPIRATION CYTOLOGY OF RETROPERITONEAL GANGLIONEUROBLASTOMA - A CASE REPORT
}

Rajshekhar S. J, S. S. Devarmani, S. K. Andola, Ashraf A. Z.

1. Professor, Department of Pathology, M. R. Medical College, Gulbarga, Karnataka.

2. Professor, Department of Pathology, M. R. Medical College, Gulbarga, Karnataka.

3. Professor \& HOD, Department of Pathology, M. R. Medical College, Gulbarga, Karnataka.

4. Post Graduate, Department of Pathology, M. R. Medical College, Gulbarga, Karnataka.

\section{CORRESPONDING AUTHOR}

Dr. Rajshekhar S. J.,

Department of Pathology,

M. R. Medical College, Gulbarga, Karnataka

E-mail: sai.drrsj@gmail.com

Ph: 00919035247898

ABSTRACT: Ganglioneuroblastoma is an uncommon peripheral neuroblastic tumor. They have intermediate malignant potential, in between that of neuroblastomas and ganglioneuromas. Histologically, they are considered to have intermediate malignant potential because they contain primitive neuroblasts along with mature ganglion cells. In literature, reports of this entity being diagnosed by fine needle aspiration and its cytological appearance are scarce.

KEY WORDS: Ganglioneuroblastoma, FNA, Neuroblastoma

INTRODUCTION: Ganglioneuroblastomas are malignant tumors of primitive neuroectodermal cells that show a great degree of maturation into neurons. Most commonly, it occurs in infants and children with a median age of 2 years. Its true global incidence, however, is unknown. According to the Surveillance, Epidemiology and End Results (SEER) Registry maintained by the National Cancer Institute, the annual incidence of neuroblastoma is 7.6 per $1,000,000$ population in the USA. ${ }^{4}$ These tumors have been rarely diagnosed by cytology.

CASE SUMMARY: A $3 \frac{1}{2}$ old year female child presented with abdominal distension and constipation since 2 weeks. On examination, a mass was palpable per abdomen. USG abdomen showed features of retroperitoneal mass and cystitis with neurogenic bladder. CT scan abdomen revealed features suggestive of neuroblastoma.

An USG guided FNA was performed, it revealed a scantly cellular smear with haemorrhagic background containing scattered large cells with abundant granular, eosinophilic cytoplasm and eccentrically placed round nucleus with coarse chromatin, some showing prominent nucleoli.(Fig. 1\&2) Binucleate cells were also noted. Also seen were neuroblasts having high nuclear-cytoplasmic ratio with round nucleus and stippled chromatin. Also noted were stromal cells and dispersed fibrillary material. A diagnosis of ganglioneuroblastoma was made.

A resection of the mass was performed. Grossly, the mass was firm, globular measuring $5 \times 4 \times 3 \mathrm{~cm}$ and the cut section showed an encapsulated homogenous white mass. Microscopically, the FNA findings were confirmed and further categorization as Ganglioneuroblastoma, stroma rich variant was made. (Fig 3) 
DISCUSSION: Ganglioneuroblastoma was defined as "a transitional tumor of sympathetic cell origin that contained malignant neuroblastomatous and benign ganglioneuromatous element" by Robertson in $1915 .{ }^{1}$ It is a primary malignant tumour of the sympathetic nervous system. ${ }^{2}$ It contains primitive neuroblast and mature ganglion cell component; and considered to have an intermediate malignant potential as compared to benign ganglioneuroma and malignant neuroblastoma. $^{3}$ It can arise anywhere along the sympathetic nervous system. ${ }^{4}$ Ganglioneuroblastomas occur with equal frequency in both the genders and most commonly in babies and in young children. ${ }^{4}$ More than $90 \%$ of cases are reported in children younger than 5 years of age, with a spike at 18 months. ${ }^{2}$ It is the third most common childhood malignancy after leukemia and brain tumors, and is the commonest solid extracranial tumor among children. ${ }^{4}$ It is very uncommon in the adult population, with fewer than 40 cases reported in the literature. ${ }^{2}$ These tumors are found most commonly in the adrenal medulla (35\%), retroperitoneum (30$35 \%)$, posterior mediastinum (20\%), neck (1-5\%), and pelvis (2-3\%). Other locations, such as the thymus, lung, kidney, anterior mediastinum, stomach, and cauda equina, are affected less frequently and considered unusual. ${ }^{5}$ The most common presentation is abdominal distension, pain and neurologic symptoms due to severe nerve root compression and sometimes Horner's syndrome (ptosis, myosis, and ipsilateral anhydrosis). ${ }^{3}$

Ganglioneuroblastomas have a propensity for secreting catecholamines, and approximately 90-95\% actively secretes vinylmandelic acid (VMA) and homovanillic acid (HVA). ${ }^{5}$ In addition, more mature tumors may contain vasoactive intestinal peptide (VIP) producing ganglion cells. Elevated levels of VIP can produce diarrhea, hypokalemia, and acidosis. $^{5}$ FNA may yield either of the two components (ganglioneuromatous or neuroblastomatous) leading to an incorrect diagnosis of either ganglioneuroma or neuroblastoma. Therefore, aspiration from multiple sites is recommended before giving a diagnosis on FNA. ${ }^{3}$ Rhabdomyosarcoma is the most common soft tissue sarcoma in children and should be kept in mind as a differential diagnosis. ${ }^{3}$ Also proliferative fascitis and myositis may show ganglion-like cells and should be considered. ${ }^{3}$ Ancillary procedures like preparation of cell block and immunohistochemistry may also aid in accurate diagnosis on FNA. ${ }^{3}$ Histological confirmation is required for definitive diagnosis. The most common site for metastasis in ganglioneuroblastomas is bone, which may mean patients present with limping and unexplained irritability (Hutchinson's syndrome). Also metastasis may occur to liver and skin producing "Pepper Syndrome" and "Blueberry muffin syndrome" respectively. ${ }^{6}$ The prognosis for patients with localized disease and younger age is better. Even the outcome of favourable histology or less aggressive neuroblastomas is worse in older children. ${ }^{4}$ In adults, prognosis depends on surgical margin resection. ${ }^{2}$ Differential markers for distinguishing ganglioneuromas from ganglioneuroblastomas at present are not available; immunohistochemical stains with antibodies such as neurofilament, synaptophysin, chromogranin, S-100 and LCA are generally positive in both ganglioneuromas and ganglioneuroblastomas. ${ }^{7}$ Complete excision remains the mainstay of therapy for localized tumors. ${ }^{4}$ Adjuvant chemotherapy and radiotherapy may also be used.

CONCLUSION: Ganglioneuroblastoma is an intermediate form between neuroblastoma (Immature form) and ganglioneuroma (Mature form). They have a better prognosis than neuroblastoma. This case highlights the utility of FNA as a safe, rapid and cost-effective modality in the diagnosis of ganglioneuroblastoma and differentiating it from other childhood tumors. Further categorization by histopathology would help in determining the prognosis. 


\section{REFERENCES:}

1. Okamatsu C, London WB, Naranjo A, Hogarty MD, Gastier-Foster JM, Look AT, LaQuaglia M, Maris JM, Cohn SL, Matthay KK, Seeger RC, Saji T, Shimada H. Clinicopathological characteristics of ganglioneuroma and ganglioneuroblastoma: a report from the CCG and COG. Pediatr Blood Cancer 2009 October;53(4):563-569.

2. Peycru T, Guiramand J,Tardat E, Savoie PH, Avaro JP, Balandraud P. Nodular ganglioneuroblastoma in adults. Can J Surg August 2009; 52(4): E111-113.

3. Choudhury M, Singh S, Goel RK. Ganglioneuroblastoma presenting as a subcutaneous lump diagnosed by fine needle aspiration. Indian J Pathol and Microbiol July- Sep 2011;54(3):614-615.

4. Fatimi SH, Bawany SA, Ashfaq A. Ganglioneuroblastoma of the posterior mediastinum: a case report. Journal of Medical Case Reports 2011;5:322.

5. Ambreen Moatasim et al. Presacral Ganglioneuroblastoma - A tumor at an unusual site. International J Pathol 2007;5(1):31-33.

6. Lucky AW, McGuire J, Komp DM. Infantile neuroblastoma presenting with cutaneous blanching nodules. J Am Acad Dermatol 1982;6:389-391.

7. Aoyama C, Qualman SJ, Regan M, Shimada H. Histopathologic features of composite ganglioneuroblastoma - Immunohistochemical distinction of the stromal component is related to prognosis. Cancer 1990;65:255-264.

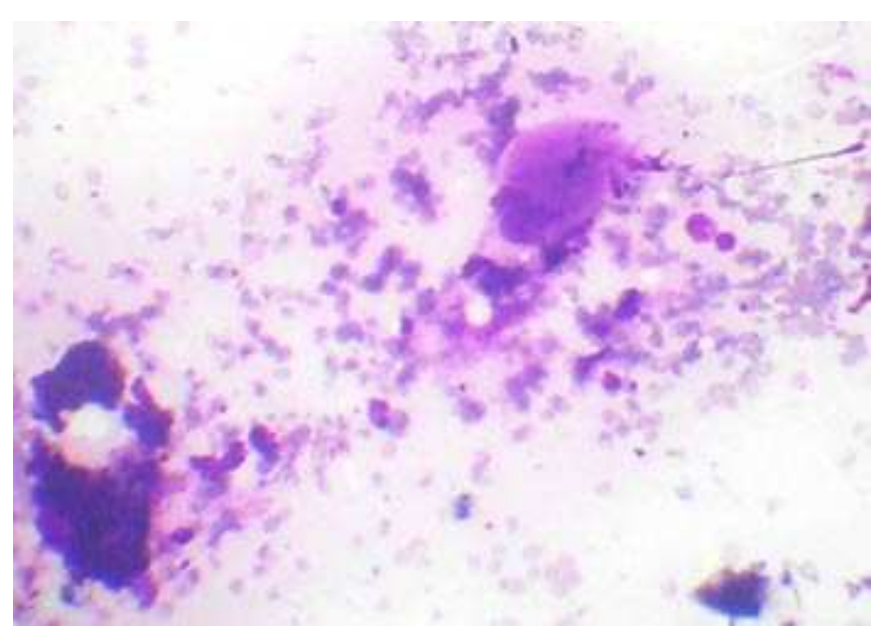




\section{CASE REPORT}
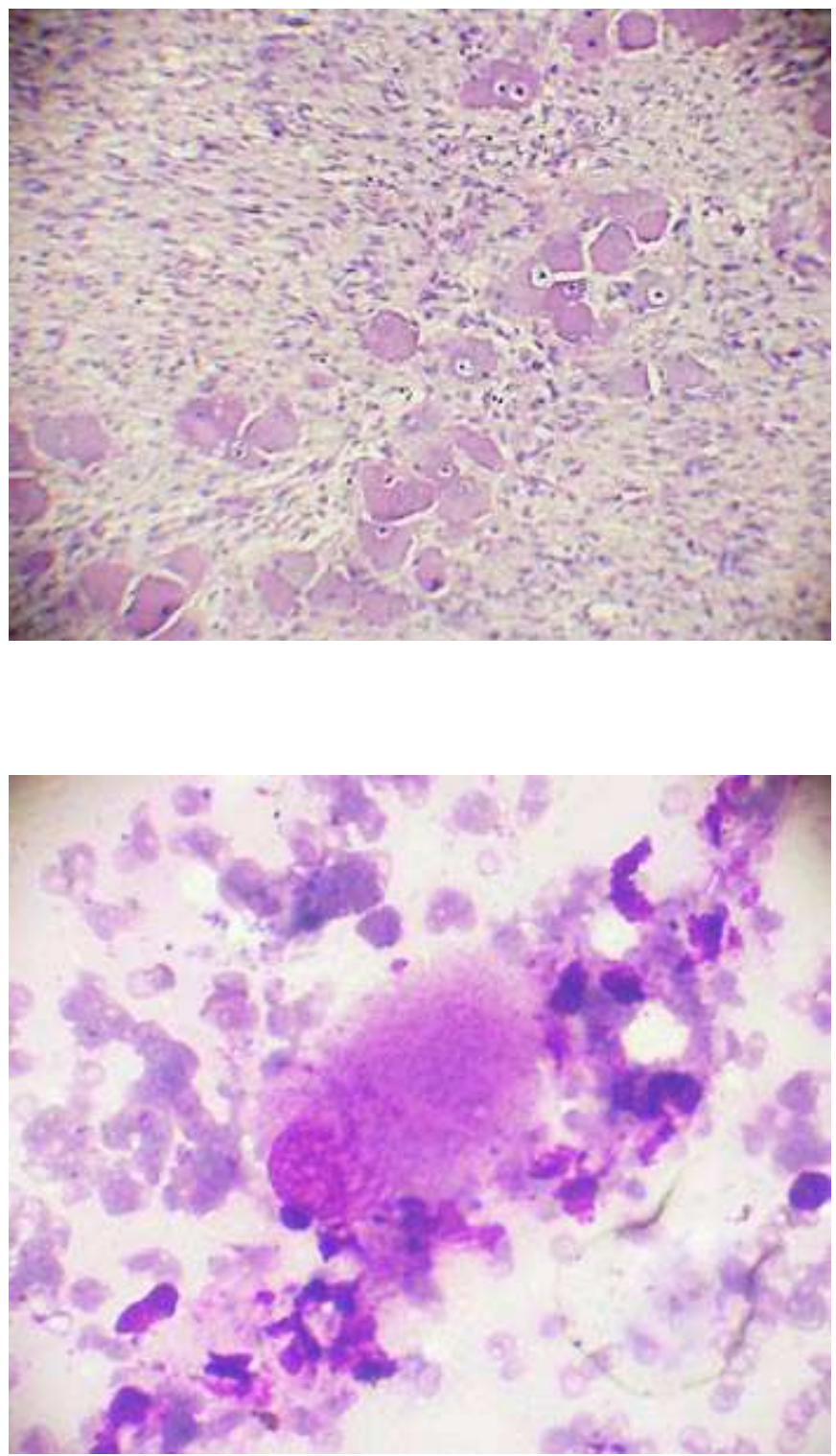